\title{
Alternating Model Trees
}

\author{
Eibe Frank \\ Department of Computer \\ Science \\ University of Waikato \\ Hamilton, New Zealand \\ eibe@cs.waikato.ac.nz
}

\author{
Michael Mayo \\ Department of Computer \\ Science \\ University of Waikato \\ Hamilton, New Zealand \\ mmayo@cs.waikato.ac.nz
}

\author{
Stefan Kramer \\ Institute of Computer Science \\ University of Mainz \\ Mainz, Germany \\ kramer@informatik.uni- \\ mainz.de
}

\begin{abstract}
Model tree induction is a popular method for tackling regression problems requiring interpretable models. Model trees are decision trees with multiple linear regression models at the leaf nodes. In this paper, we propose a method for growing alternating model trees, a form of option tree for regression problems. The motivation is that alternating decision trees achieve high accuracy in classification problems because they represent an ensemble classifier as a single tree structure. As in alternating decision trees for classification, our alternating model trees for regression contain splitter and prediction nodes, but we use simple linear regression functions as opposed to constant predictors at the prediction nodes. Moreover, additive regression using forward stagewise modeling is applied to grow the tree rather than a boosting algorithm. The size of the tree is determined using cross-validation. Our empirical results show that alternating model trees achieve significantly lower squared error than standard model trees on several regression datasets.
\end{abstract}

\section{Categories and Subject Descriptors}

I.2.6 [Artificial Intelligence]: Learning

\section{General Terms}

Algorithms, Experimentation, Performance

\section{Keywords}

Regression, alternating model trees

\section{INTRODUCTION}

Alternating decision trees [5] provide the predictive power of decision tree ensembles in a single tree structure. They are a variant of option trees 3, 9, i.e., decision trees augmented with option nodes, and grown using boosting. Existing approaches for growing alternating decision trees focus on classification problems. In this paper, we investigate alternating

Permission to make digital or hard copies of all or part of this work for personal or classroom use is granted without fee provided that copies are not made or distributed for profit or commercial advantage and that copies bear this notice and the full citation on the first page. To copy otherwise, to republish, to post on servers or to redistribute to lists, requires prior specific permission and/or a fee.

SAC'15 April 13-17, 2015, Salamanca, Spain.

Copyright 2015 ACM 978-1-4503-3196-8/15/04 ...\$15.00.

http://dx.doi.org/10.1145/2695664.2695848 model trees for regression, inspired by work on model trees for regression [15] and work on growing alternating decision trees for multi-class classification 8].

Model trees for regression are decision trees with linear regression models at the leaf nodes. They were originally proposed by Quinlan [15. An open-source implementation, called $\mathrm{M} 5^{\prime}$, was presented in 18 and has proven to be successful in many practical applications, e.g., 16, 2, 14, 4, 13, 17. An example model tree grown using $\mathrm{M} 5^{\prime}$ is shown in Figure 1 The inclusion of linear regression models rather than constant predictors at the leaf nodes is essential: standard regression trees with constant predictors produce much less accurate predictions than model trees 15.

M5 trees are grown using the standard top-down approach for growing decision trees. Once an unpruned tree has been grown, multiple linear regression models are placed at each node of the tree. Following this, the tree is pruned, potentially replacing large subtrees by a single linear regression model. Finally, the linear regression models along the path from the root node of the tree to each leaf node are combined into a single linear regression model at the leaf node using a "smoothing" process that produces a linear combination of linear regression models. For further details, see [15, 18.

The learning algorithm for alternating model trees we present in this paper grows trees using additive regression. In [8], alternating decision trees for multi-class classification are grown using additive logistic regression, a statistical variant of boosting. The process we apply in this paper is very similar, applying forward stagewise additive modeling for minimization of squared error rather than maximization of multinomial likelihood. The key difference of our method is that we use (simple) linear regression models instead of constant predictors in the tree so that we can obtain competitive predictive performance on regression problems. Moreover, to reduce computational complexity and data fragmentation, splits on numeric attributes at splitter nodes are restricted to the median value of each attribute rather than arbitrary points identified by a split selection criterion.

\section{ALTERNATING MODEL TREES}

We assume that we have a training dataset $D$ with $n$ instances consisting of $m$ real-valued attributes and a realvalued target attribute. The learning algorithm is provided with $n$ instances $\left(\overrightarrow{x_{i}}, y_{i}\right)$, with $1 \leq i \leq n$, where $\overrightarrow{x_{i}}$ is a vector of attribute values and $y_{i}$ is the associated target value. The task is to learn a model such that, given a new $\vec{x}$, this model can be used to predict the corresponding value $y$. In this paper, we assume that the goal is to minimize the squared 


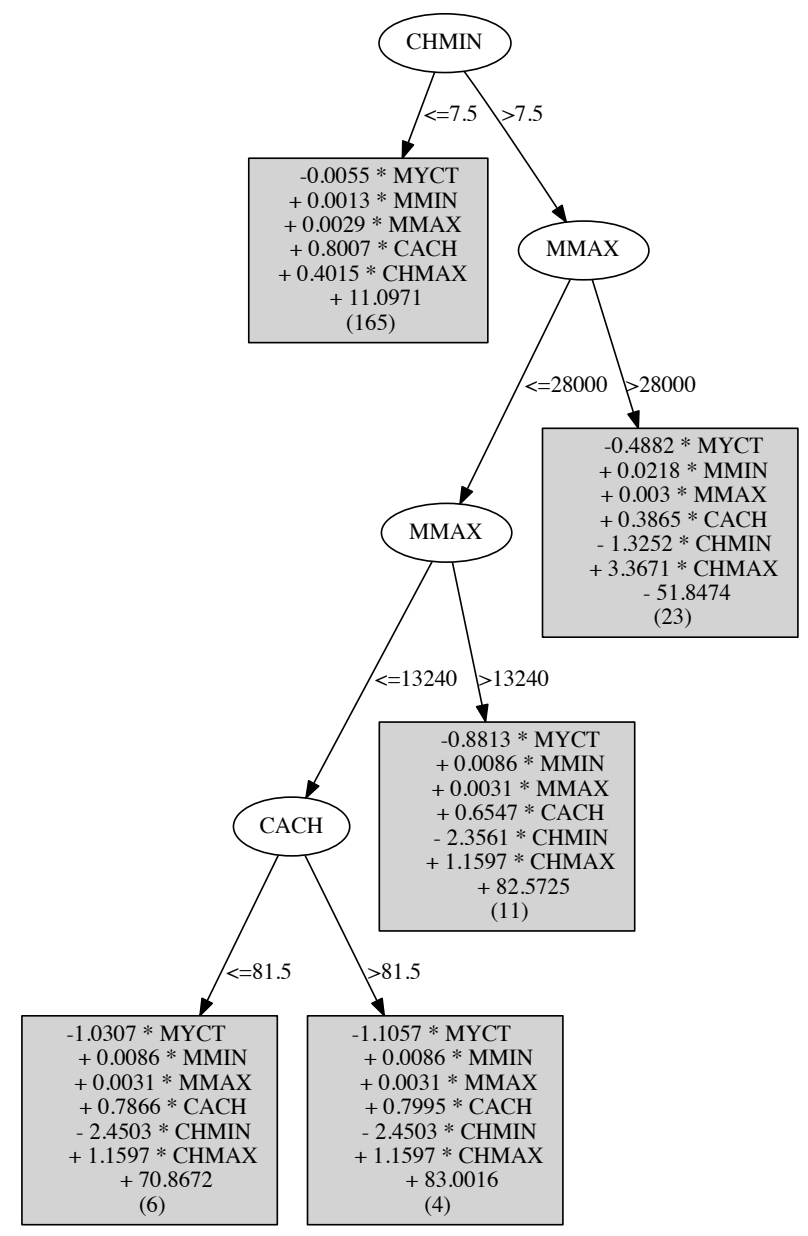

Figure 1: $\mathrm{M5}^{\prime}$ model tree for machine-cpu data

error of the model between the predicted and actual target values. Furthermore, we assume that the data is independently and identically distributed.

\subsection{Forward Stagewise Additive Modeling}

Our method for learning additive model trees applies the basic algorithm for additive regression using forward stagewise additive modeling [6]. The strategy is to iteratively correct the predictions of an additive model $F_{j}(\vec{x})$ by taking the residual errors it makes on the training data - the differences between the actual and predicted target values - and applying a base learner that learns to predict those residuals. The model $f_{j+1}(\vec{x})$ output by the base learner is then appended to the additive model and the residuals are updated for the next iteration.

More precisely, we fit a model consisting of $k$ base models,

$$
F_{k}(\vec{x})=\sum_{j=1}^{k} f_{j}(\vec{x})
$$

in a forward stagewise manner so that the squared error

$$
\sum_{i=1}^{n}\left(F_{k}\left(\vec{x}_{i}\right)-y_{i}\right)^{2}
$$

over the $n$ training examples $\left(\vec{x}_{i}, y_{i}\right)$ is minimized.
Input: $D$ : training data, $B L$ : base learner, $I:$ number of iterations to perform, $\lambda$ : shrinkage parameter $\in(0,1]$

Output: $L$ : list containing ensemble predictor

$L:=$ empty list

L.add $\left(\frac{1}{n} \sum_{(\vec{x}, y) \in D} y\right)$

foreach $(\vec{x}, y) \in D$ do

$(\vec{x}, y):=(\vec{x}, y-L . f i r s t() \cdot p r e d i c t i o n(\vec{x}))$

end

while $|L| \leq I$ do

L.add $(\bar{B}$ L.buildModel $(D))$

foreach $(\vec{x}, y) \in D$ do

I $(\vec{x}, y):=(\vec{x}, y-\lambda \times$ L.last ()$\cdot \operatorname{prediction}(\vec{x}))$

end

end

return $L$

Algorithm 1: Additive regression using forward stagewise additive modeling

In a particular iteration $k$, we fit a base model $f_{k}(\vec{x})$ (e.g., a regression stump) to the residuals $y-F_{k-1}(\vec{x})$ remaining from the $k-1$ previous iterations. We fit these residuals by minimizing the squared error between them and the base model's predictions $f_{k}(\vec{x})$.

In this manner, we ensure that the squared error of the additive model is minimized, assuming the existing part of the model $F_{k-1}(\vec{x})$ can no longer be modified:

$$
\begin{aligned}
& \sum_{i=1}^{n}\left(\left(y_{i}-F_{k-1}\left(\vec{x}_{i}\right)\right)-f_{k}\left(\vec{x}_{i}\right)\right)^{2} \\
= & \sum_{i=1}^{n}\left(y_{i}-\left(F_{k-1}\left(\vec{x}_{i}\right)+f_{k}\left(\vec{x}_{i}\right)\right)\right)^{2}=\sum_{i=1}^{n}\left(y_{i}-F_{k}\left(\vec{x}_{i}\right)\right)^{2}
\end{aligned}
$$

This is thus a greedy algorithm to fit an additive regression model by minimizing squared error.

Pseudo code for the algorithm is shown in Algorithm 1 It has three arguments: the base regression learner to use, the number of iterations to perform, and the value of the shrinkage parameter used to control overfitting. The algorithm first calculates the mean target value and makes this predictor the first element of the additive model. Subtracting the mean establishes the initial set of residuals. In each iteration of additive regression, the base regression scheme is applied to learn to predict the residuals. Subsequently, the predicted residuals are subtracted from the actual residuals to establish the new set of residuals for the next iteration. This is repeated for the given number of iterations.

The shrinkage parameter is a value in $(0,1]$. (A value of 1 means that no shrinkage is performed.) In the algorithm, the predictions of the base learner are multiplied by the shrinkage value when the residuals are calculated. When the shrinkage value is reduced by the user, the predictions of each base model are dampened before being included in the additive model. Because the mean predictor is the first element in the model, and is not affected by the shrinkage parameter, the predictions of the additive model are therefore shrunk towards the mean. This makes it possible to perform regularization of the model.

The prediction step is shown in Algorithm 2 It is straightforward: the predictions of all base models are simply multiplied by the shrinkage value and added to the mean target value from the training data. This is then the final prediction of the additive model. 
Input: $\vec{x}$ : the instance to make a prediction for, $L$ : list of regressors

Output: $y$ : the prediction

$y=L . f i r s t() \cdot p r e d i c t i o n(\vec{x})$

foreach $M \in L$ do

if $M \neq L$.first() then

$y=y+\lambda \times M$.prediction $(\vec{x})$

end

end

return $y$

Algorithm 2: Generating a prediction using the additive regression model

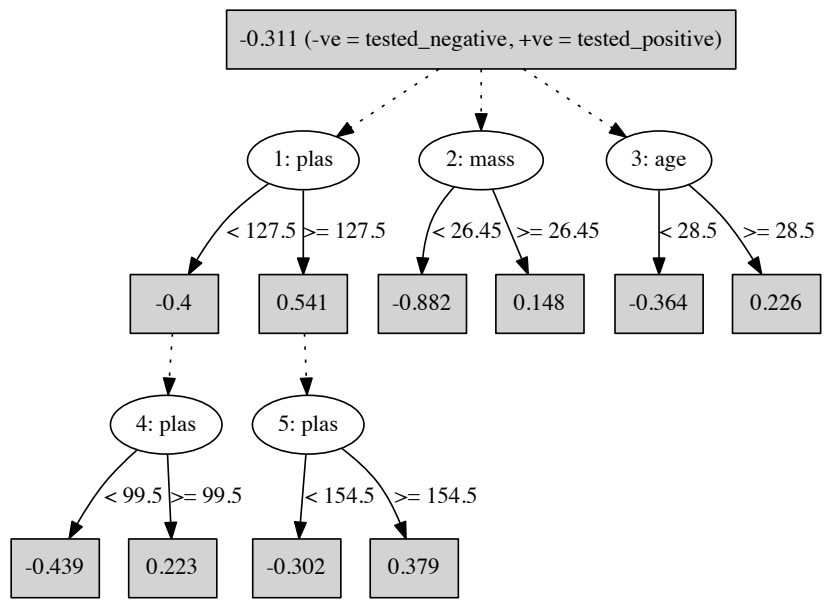

Figure 2: Alternating decision tree with five splitter nodes grown from pima-indians diabetes data

\subsection{Constructing Alternating Model Trees}

An alternating decision tree consists of two types of nodes: splitter nodes and prediction nodes [5]. In the classification case, it is grown by adding one splitter node and two prediction nodes in each iteration of the boosting algorithm that generates the tree. A splitter node is the same as an internal node in a standard decision tree and divides the data based on a chosen attribute-value test. Prediction nodes contain numeric scores that are used to make a prediction. When an instance is to be classified, it is routed down all applicable paths in the tree and the numeric scores of all visited prediction nodes are summed to yield the final prediction. For example, in the two-class classification case, the sum may be positive, in which case the positive class is predicted; otherwise, the negative class is predicted.

Figure 2 shows an alternating decision tree grown using the algorithm from [5], for the two-class pima-indians diabetes data available from the UCI repository of datasets [1]. Five boosting iterations where performed to grow this tree so that five splitter nodes are included. The root node has three prediction nodes attached to it. When a prediction is to be made, all three options need to be considered.

Consider an instance that has the following values for the attributes used in the tree: plas $=130$, mass $=27.1$ and age $=30$. The score for this instance involves five prediction nodes and would be $-0.311+0.541+0.148+0.226-0.302=$ $0.302>0$. Hence, tested_positive would be the class predicted for this instance.

An alternating decision tree is an option tree because each prediction node can have multiple splitter nodes as successors (or none, in which case it is a leaf node). In each boosting iteration, a prediction node in the current version of the alternating tree is identified, and a splitter node, with two new prediction nodes attached to it, is added to it as a successor. The particular prediction node chosen for this and the split applied at the splitter node are selected such that the loss function of the boosting algorithm is minimized.

We can view an alternating decision tree as a collection of if-then rules, where each path in the tree corresponds to a rule. In each iteration of the boosting algorithm, a particular rule is chosen for extension by an additional attribute-value test (implemented by the splitter node), and two extended versions of the rule are added to the rule set. Thus, we can view the process of growing an alternating decision tree as an application of boosting with a base learner that grows restricted if-then rules: the two new rules added in a particular iteration consist of a prefix that already exists in the tree, one new test is added to both rules, and the new rules include appropriately chosen values on the right-hand side.

We can easily adapt this method for growing an alternating decision tree so that it can be applied to regression problems. Given the algorithm for generating an additive model for regression discussed above, we can grow an alternating decision tree for regression by applying an appropriate base learner. In each iteration of additive regression, we add one splitter node and two prediction nodes to the tree. The parameters of these nodes and their location in the alternating tree are chosen so that squared error is minimized.

However, because we want to grow alternating model trees, we do not simply add constant values at the prediction nodes, but rather apply simple linear regression functions ${ }^{1}$ A splitter node is constructed by considering splits on all available attributes in the data, splitting on the median attribute value for the corresponding data that reaches the node to be split, and fitting two simple linear regression models to the subsets created by the split. The two attributes for the simple linear regression models are chosen so that squared error is minimized.

Of all available splits, one for each attribute and existing prediction node, the split that minimizes the squared error of the alternating tree is chosen, and the corresponding two linear regression models are placed at the two new prediction nodes that are attached to the new splitter node. Note that the two simple linear regression models for the two subsets of data generated by the median-based split can be based on different attributes. For each subset, all attributes are considered, and that particular attribute is chosen whose simple linear regression model minimizes squared error.

Figure 3 shows an example model tree for the machine-cpu data. Five iterations of additive regression where performed so the tree contains five splitter nodes and 11 prediction nodes, including the root node which simply predicts the mean target value from the training data. The lowest level of the tree has two splitter nodes that are attached to the same prediction node. Hence, if an instance reaches this prediction node, both successors need to be visited and a prediction obtained from both of them. The numbers in

\footnotetext{
${ }^{1}$ In contrast to a multiple linear regression model as used in M5, a simple linear regression model is a regression model based on a single, appropriately chosen, predictor attribute. Simple linear regression models are also used in the model tree inducers presented in [10, 11.
} 


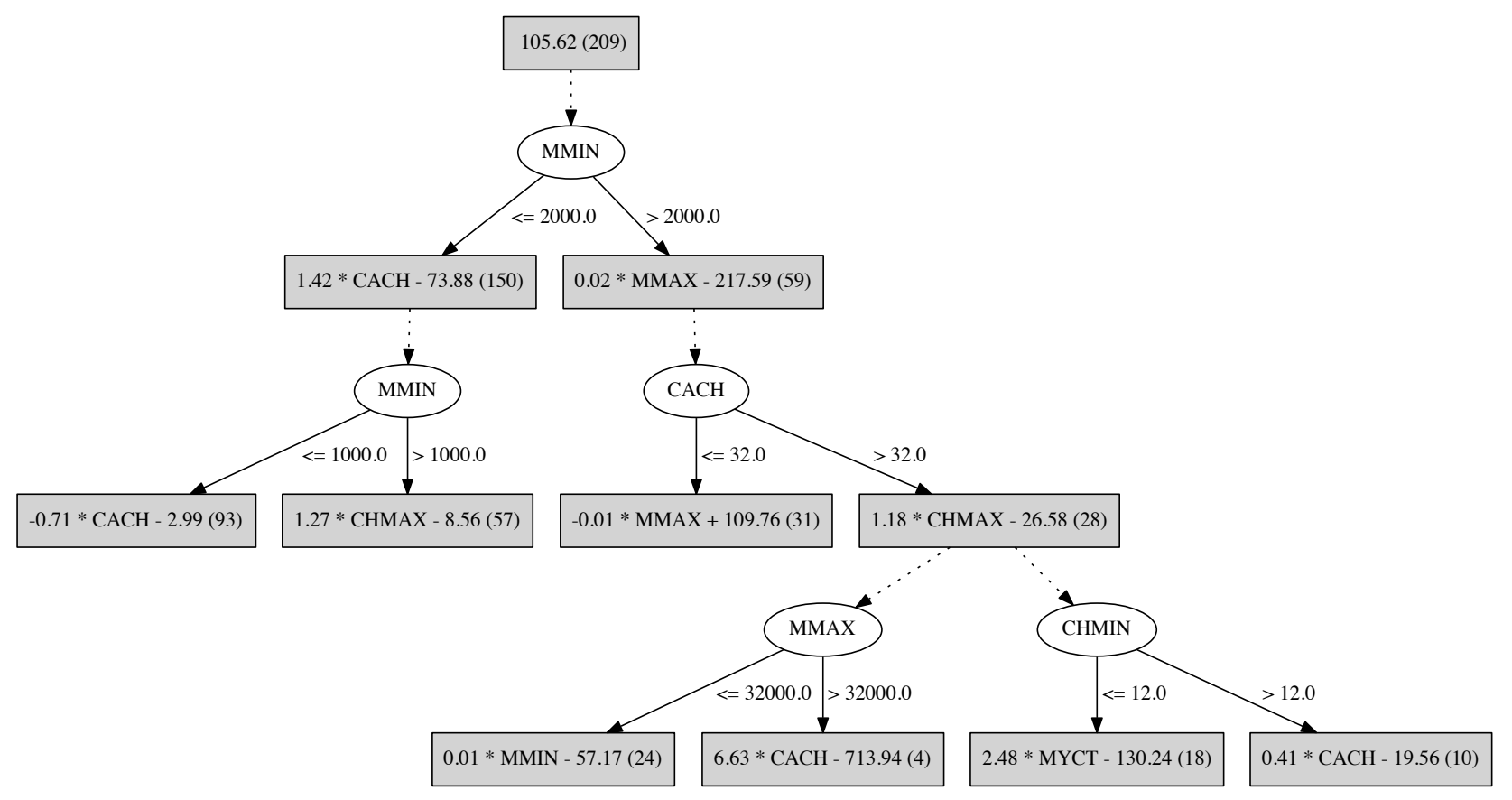

Figure 3: Alternating model tree for machine-cpu data

brackets show the number of training instances that reach a particular prediction node. With continuous data, they are equal for the two successors of a prediction node, because the median value is used for splitting, but this data has discrete values and hence the splits are not balanced.

Algorithm 3 has the pseudo code for growing the alternating model tree. It employs three types of objects:

- SplitterNode objects that contain a reference to their parent prediction node; the score - reduction in squared error - they achieve; the parameters for the split (attribute index and value of split point); and references to the two successor prediction nodes.

- PredictionNode objects that contain a reference to the regression model employed; the data associated with the node; and a list of successors, holding references to splitter nodes that are its successors.

- $S L R$ objects that perform simple linear regression based on the data for a given attribute and the target; they also contain the sum of squared errors (SSE) achieved and are able to yield a prediction for a new instance.

The algorithm maintains a list of prediction nodes that initially only contains the predictor of the mean. The target values are also centered, just as in Algorithm 1 1 Then, a given user-specified number of iterations is performed, and in each iteration, a new Splitter Node is added to a selected prediction node. To select this node, the algorithm iterates through all PredictionNode objects already in the list. For each prediction node, the current sum of squared errors (i.e. sum of squared residuals/target values) associated with the data pertaining to this node is established first, so that the reduction of squared error that is achieved by a split attached to this node can be computed later. Then, all attributes are evaluated for a median-based split: for each split, the data is divided into two subsets and the best linear model (lowest SSE) is found for each subset. If the current split yields a larger reduction in SSE than the previous best one, the information for the splitter node is updated.

Once the best Splitter Node has been constructed, it is attached to its parent node, and its two attached prediction nodes are added to the global list of prediction nodes so that they become available for selection in the next iteration of the algorithm. After this, the residuals/target values are updated for the two subsets of data associated with the split. Note that we assume that only one copy of each instance $(\vec{x}, y)$ in $D$ is maintained in memory, and that this copy is modified when the residuals are updated, i.e., we use $m u$ table tuples. Hence, the effect of the update is global and affects the data to be considered in the next iteration at all applicable prediction nodes in the tree. Hence, the process is consistent with the algorithm for additive regression.

Algorithm 4 shows the pseudo code for making predictions using the tree. It is given the root node of the tree and maintains a queue of prediction nodes to include in the prediction. The root node is the first element in the queue. As it predicts the mean target value from the training data, no shrinkage is applied to its prediction. If the queue is not empty, the first prediction node is popped off the queue and its prediction is added to the running sum. Then, all splitter nodes that are successors of the current prediction node are evaluated, and the appropriate successor of each splitter 
Input: $D$ : training data, $I$ : number of iterations to perform

Output: $R$ : The root node of the model tree

$L:=$ empty list

L.add $\left(\right.$ Prediction Node $\left(\right.$ model $=\frac{1}{n} \sum_{(\vec{x}, y) \in D} y$, data $=D$, successors $=$ empty list)

foreach $(\vec{x}, y) \in D$ do $/ *$ initialize residuals $* /$

$\mid(\vec{x}, y):=(\vec{x}, y-$ L.first (). model.prediction $(\vec{x}))$

end

while $|L| \leq I$ do $/ *$ for given number of iterations */

$S:=$ Splitter Node $($ parent $=$, score $=-\infty$, attribute $=$, splitPoint $=$, left $=$, right $=$ )

foreach $P \in L$ do $\quad / *$ for each prediction node */ $S S E:=\sum_{(\vec{x}, y) \in P \text {.data }} y^{2}$

/* consider each attribute for splitting */ foreach $j, 1<j<m$ do

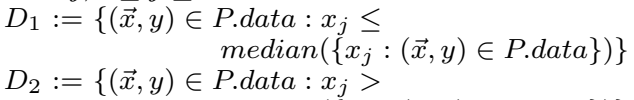

Algorithm 3: Growing an alternating model tree
Input: $\vec{x}$ : the instance to make a prediction for, $R$ : root node of alternating model tree

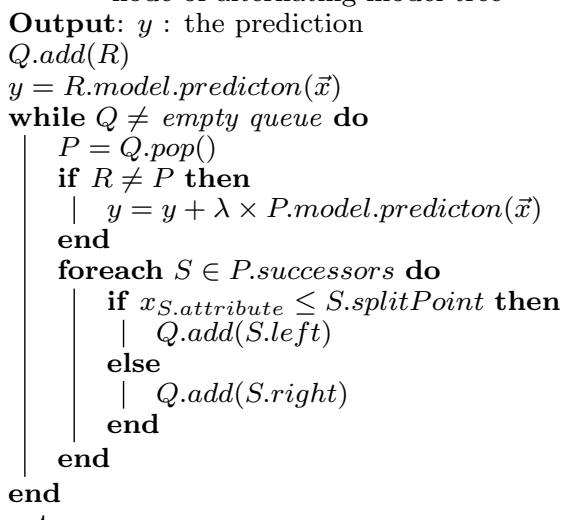

return $y$

Algorithm 4: Generating a prediction using the alternating model tree

node is added to the queue of prediction nodes. Once the queue becomes empty, the final sum is returned.

\section{CHOOSING A TREE SIZE}

The algorithm as stated requires the user to specify the number of iterations to perform. Choosing an appropriate tree size is clearly important to obtain low squared error on new data. In practice, it is common to use cross-validation to choose the number of iterations for boosting-like algorithms. This can be done efficiently because it is unnecessary to grow the entire additive model from scratch when evaluating a particular number of iterations. For a $k$-fold cross-validation, $k$ alternating decision trees can be maintained simultaneously in memory. Once the current set of trees of size $I$ have been evaluated, an additional execution of the main loop in Algorithm 3 can be performed for each of the trees to get the trees corresponding to size $I+1$.

Rather than stopping iterations immediately when the cross-validated squared error no longer decreases, we continue performing iterations unless no better minimum can be found in the next 50 iterations from the current minimum squared error. This is to prevent premature stopping due to variance in the cross-validation estimates.

This algorithm is well-suited for modern multi-core machines because cross-validation can be easily parallelized. The constant multiplier in runtime imposed by cross-validation is thus less problematic. Note that, for a given number of iterations $I$, the runtime of Algorithm 3 is $O\left(I^{2} m^{2} n\right)$ in the worst case. The worst case occurs when all splitter nodes are attached to the root node of the tree and only minimal splitting of the data occurs. This also yields the worst case for the prediction step in Algorithm 4 in which all splitter nodes need to be evaluated, so its complexity is $O(I)$.

\section{EXPERIMENTAL RESULTS}

In this section, we present empirical results obtained on Luis Torgo's collection of benchmark datasets for regression ${ }^{2}$ considering root mean squared error and tree size. They vary in size from less than 100 instances to more than

$\sqrt[2]{\text { http://www.dcc.fc.up.pt/ Itorgo/Regression/ }}$ DataSets.html 


\begin{tabular}{|c|c|c|c|c|c|c|}
\hline Dataset & $\mathrm{M}^{\prime}$ & $\begin{array}{l}\text { AMT } \\
(\lambda=1)\end{array}$ & & $\begin{array}{c}\text { AMT } \\
(\lambda=0.5)\end{array}$ & $\begin{array}{c}\text { AMT } \\
(\lambda=0.1)\end{array}$ & \\
\hline 2dplanes & $22.7 \pm 0.4$ & $23.4 \pm 0.6$ & o & $23.1 \pm 0.4$ & $23.0 \pm 0.4$ & \\
\hline abalone & $66.0 \pm 2.9$ & $67.6 \pm 2.5$ & $\circ$ & $67.9 \pm 2.9$ & $67.7 \pm 2.4$ & 0 \\
\hline ailerons & $39.7 \pm 1.0$ & $39.7 \pm 1.2$ & & $38.9 \pm 1.1$ & $38.5 \pm 1.1$ & - \\
\hline autoMpg & $34.9 \pm 6.2$ & $36.3 \pm 6.2$ & & $37.0 \pm 8.5$ & $35.0 \pm 6.1$ & \\
\hline & $37.8 \pm 10.2$ & $42.3 \pm 17.0$ & & $36.9 \pm 14.5$ & $34.3 \pm 11.8$ & \\
\hline & $67.4 \pm 2.2$ & $67.9 \pm 2.4$ & & $67.6 \pm 2.3$ & $67.4 \pm 2.3$ & \\
\hline bank8FM & $.0 \pm 0.7$ & $19.9 \pm 0.7$ & & $19.5 \pm 0.7$ & $19.5 \pm 0.7$ & - \\
\hline cal-housing & $48.5 \pm 2.8$ & $42.8 \pm 1.7$ & & $42.7 \pm 2.3$ & - $\quad 42.2 \pm 2.3$ & - \\
\hline $\mathrm{cpu}-$ & $14.8 \pm 2.0$ & $13.3 \pm 1.4$ & - & $13.6 \pm 5.0$ & $12.7 \pm 1.5$ & - \\
\hline & \pm 1.8 & $15.8 \pm 1.4$ & - & $15.7 \pm 4.4$ & $15.1 \pm 1.3$ & - \\
\hline delta- & $.3 \pm 2.0$ & $54.0 \pm 2.1$ & & $53.7 \pm 2.1$ & $54.3 \pm 3.4$ & \\
\hline delta-elevators & $60.0 \pm 1.4$ & $59.8 \pm 1.4$ & & $59.8 \pm 1.4$ & $60.0 \pm 1.7$ & \\
\hline diak & $91.7 \pm 41.5$ & $106.2 \pm 54.2$ & & $93.7 \pm 50.5$ & $95.8 \pm 40.4$ & \\
\hline ors & $32.3 \pm 1.2$ & $32.2 \pm 2.4$ & & $31.8 \pm 1.9$ & $31.7 \pm 1.5$ & \\
\hline fried & $27.8 \pm 0.5$ & $20.2 \pm 0.3$ & - & $20.2 \pm 0.3$ & $20.2 \pm 0.3$ & - \\
\hline house- $16 \mathrm{H}$ & $68.2 \pm 4.5$ & $70.8 \pm 13.8$ & & $67.4 \pm 5.9$ & $66.5 \pm 7.0$ & \\
\hline & \pm 4.2 & $60.1 \pm 3.2$ & & $59.3 \pm 3.2$ & $59.4 \pm 3.5$ & \\
\hline & $40.8 \pm 11.3$ & $60.4 \pm 45.9$ & & $57.9 \pm 37.8$ & $52.6 \pm 31.4$ & \\
\hline kin8nm & $60.8 \pm 2.2$ & $44.4 \pm 1.5$ & & $41.4 \pm 1.3$ & $40.7 \pm 5.1$ & - \\
\hline achine-cpu & $39.4 \pm 17.6$ & $46.9 \pm 21.4$ & & $36.5 \pm 18.2$ & $31.8 \pm 15.7$ & \\
\hline & $1.3 \pm 0.1$ & $0.1 \pm 0.0$ & - & $0.6 \pm 3.7$ & $0.1 \pm 0.0$ & - \\
\hline & 15.6 & $23.1 \pm 5.4$ & ० & $19.2 \pm 5.6$ & $15.7 \pm 1.3$ & \\
\hline ma32H & 27.1 & $67.2 \pm 18.0$ & & $44.5 \pm 25.1$ & $24.5 \pm 12.37$ & \\
\hline $8 \mathrm{NH}$ & $56.9 \pm 1.5$ & $56.7 \pm 1.5$ & & $57.0 \pm 1.5$ & $57.3 \pm 1.5$ & \\
\hline pyri & $67.0 \pm 27.6$ & $100.0 \pm 35.0$ & & $82.6 \pm 29.3$ & $73.3 \pm 29.5$ & \\
\hline rvo & $35.9 \pm 14.7$ & $33.0 \pm 18.4$ & & $32.2 \pm 16.8$ & $30.5 \pm 16.7$ & \\
\hline & & $15.6 \pm 3.3$ & & $13.3 \pm 2.1$ & $12.2 \pm 1.7$ & - \\
\hline & & $98.8 \pm 18.7$ & & $90.0 \pm 19.5$ & $84.7 \pm 14.4$ & \\
\hline wisconsin & $98.5 \pm 9.7$ & $100.2 \pm 6.7$ & & $97.3 \pm 5.6$ & $98.0 \pm 5.3$ & \\
\hline
\end{tabular}

Table 1: Root relative squared error on benchmark datasets, estimated using $10 \times 10$-fold cross-validation

40,000 instances. The number of predictor attributes varies between two and 60 .

We implemented our algorithm for learning alternating trees in Java using the WEKA framework [7. Just as in $\mathrm{M} 5^{\prime}$ in WEKA, our implementation converts nominal attributes into binary numeric attributes using the supervised NominalToBinary filter and replaces missing values by the attribute's corresponding mean value. Our experiments were performed using Oracle's Java 1.7.0_51 for Linux, on computers with four-core Intel Core i7-2600 CPUs and 16GB of RAM. To choose an appropriate tree size, we implemented an IterativeClassifierOptimizer, which chooses the number of iterations for growing the tree efficiently using the method described in the preceding section. We used 10-fold cross-validation to choose the number of iterations, parallelized using five threads.

Table 1 shows root relative squared error, obtained with the WEKA Experimenter [7] and $10 \times 10$-fold cross-validation. Standard deviations for the 100 results are also shown. Note that this involved running the basic alternating model tree algorithm 1,000 times: for each split of the outer $10 \times 10$-fold cross-validation, the inner cross-validation implemented by IterativeClassifierOptimizer was run separately on the training data for that split. This was to avoid parameter tuning (i.e. selection of tree size) based on the test data, thus biasing results. The table shows root relative squared error, which is root mean squared error of the scheme being evaluated divided by the root mean squared error obtained when predicting the mean target value from the training data. It has been scaled by multiplying it with 100 . Values smaller than 100 indicate an improvement on the mean.

The left-most column shows estimated error for the model tree learner $\mathrm{M} 5^{\prime}$, as implemented in WEKA. The other three columns are for alternating model trees, run with three different values for the shrinkage parameter: 1 (no shrinkage), 0.5 , and 0.1 . To establish statistical significance of observed differences in estimated error, we used the paired corrected resampled $t$-test 12 at the 0.05 significance level. A hollow circle indicates a statistically significant degradation with respect to $\mathrm{M} 5^{\prime}$. A filled circle indicates an improvement.

We can see that error estimates are comparable in general. However, there are several datasets where alternating model trees perform significantly better than $\mathrm{M} 5^{\prime}$ model trees, and a few where they perform significantly worse. In particular, alternating model trees perform significantly worse on the puma32H data when using $\lambda=1$ or 0.5 . Decreasing shrinkage improves error in general, in particular on the puma32H data, indicating that there is a problem with overfitting on this data. Inspection of the trees grown on various datasets shows that when $\lambda=1$, the trees grow deep very quickly, resulting in data fragmentation and increasing the potential for overfitting. As $\lambda$ is reduced, the trees become shallower.

Table 2 shows average tree size, as measured by the number of splitter/decision nodes. The results show that decreasing the shrinkage parameter increases the size of the trees, i.e. cross-validation-based model selection as implemented using IterativeClassifierOptimizer performs more iterations. Note that, just as in $\mathrm{M}^{\prime}$ trees, the linear regression models occurring along a path in an alternating model tree can be consolidated into a single multiple linear regression model stored at the leaf node associated with this path, because the sum of a set of simple linear regression models 


\begin{tabular}{l|c|ccc}
\hline Dataset & M5 $^{\prime}$ & $\begin{array}{c}\text { AMT } \\
(\lambda=1)\end{array}$ & $\begin{array}{c}\text { AMT } \\
(\lambda=0.5)\end{array}$ & $\begin{array}{c}\text { AMT } \\
(\lambda=0.1)\end{array}$ \\
\hline 2dplanes & $1.0 \pm 0.0$ & $151.9 \pm 58.3$ & $225.2 \pm 44.4$ & $844.7 \pm 124.5$ \\
abalone & $7.6 \pm 3.2$ & $8.2 \pm 2.1$ & $18.1 \pm 3.4$ & $103.1 \pm 25.2$ \\
ailerons & $5.4 \pm 1.7$ & $33.5 \pm 7.7$ & $78.4 \pm 17.9$ & $467.1 \pm 113.7$ \\
autoMpg & $2.9 \pm 1.5$ & $5.4 \pm 2.2$ & $11.3 \pm 6.3$ & $93.4 \pm 38.6$ \\
auto-price & $6.7 \pm 2.3$ & $6.2 \pm 4.7$ & $14.5 \pm 10.1$ & $81.2 \pm 34.2$ \\
bank32nh & $3.6 \pm 3.5$ & $16.8 \pm 3.5$ & $35.1 \pm 5.8$ & $260.8 \pm 53.7$ \\
bank8FM & $29.8 \pm 6.7$ & $45.1 \pm 6.8$ & $81.0 \pm 10.9$ & $444.5 \pm 63.47$ \\
cal-housing & $205.8 \pm 28.7$ & $172.6 \pm 31.0$ & $264.7 \pm 49.8$ & $927.7 \pm 222.5$ \\
cpu-act & $47.6 \pm 14.2$ & $62.0 \pm 12.9$ & $115.5 \pm 24.6$ & $462.1 \pm 135.2$ \\
cpu-small & $50.1 \pm 14.7$ & $63.1 \pm 12.3$ & $119.2 \pm 21.4$ & $495.7 \pm 148.9$ \\
delta-ailerons & $23.6 \pm 7.2$ & $17.7 \pm 4.1$ & $28.5 \pm 6.1$ & $159.8 \pm 42.3$ \\
delta-elevators & $6.2 \pm 2.4$ & $12.1 \pm 2.6$ & $26.2 \pm 4.6$ & $133.7 \pm 24.5$ \\
diabetes-numeric & $0.7 \pm 1.0$ & $2.3 \pm 1.3$ & $3.6 \pm 1.5$ & $18.0 \pm 8.9$ \\
elevators & $33.5 \pm 8.6$ & $129.1 \pm 27.6$ & $257.9 \pm 38.8$ & $1153.5 \pm 200.5$ \\
fried & $508.5 \pm 38.3$ & $57.5 \pm 6.7$ & $121.6 \pm 6.6$ & $673.2 \pm 40.5$ \\
house-16H & $181.1 \pm 48.1$ & $37.8 \pm 11.6$ & $48.6 \pm 15.4$ & $208.6 \pm 74.3$ \\
house-8L & $126.1 \pm 28.0$ & $33.5 \pm 7.2$ & $58.5 \pm 14.9$ & $247.3 \pm 93.1$ \\
housing & $12.0 \pm 5.3$ & $4.3 \pm 6.6$ & $13.0 \pm 18.6$ & $73.4 \pm 72.6$ \\
kin8nm & $108.7 \pm 18.0$ & $263.4 \pm 36.1$ & $527.4 \pm 61.1$ & $2927.8 \pm 377.7$ \\
machine-cpu & $2.7 \pm 1.5$ & $5.3 \pm 5.9$ & $12.8 \pm 7.1$ & $74.1 \pm 32.8$ \\
mv & $9.1 \pm 0.3$ & $363.6 \pm 119.9$ & $523.6 \pm 286.5$ & $2232.3 \pm 866.7$ \\
pol & $169.5 \pm 19.6$ & $89.6 \pm 24.9$ & $196.4 \pm 62.5$ & $1047.6 \pm 330.9$ \\
puma32H & $103.9 \pm 21.8$ & $86.0 \pm 27.2$ & $145.3 \pm 39.8$ & $998.3 \pm 305.24$ \\
puma8NH & $27.0 \pm 4.5$ & $16.3 \pm 1.4$ & $29.5 \pm 2.8$ & $198.4 \pm 32.8$ \\
pyrim & $1.3 \pm 1.0$ & $3.4 \pm 6.1$ & $7.5 \pm 6.7$ & $51.4 \pm 37.3$ \\
servo & $5.1 \pm 1.6$ & $13.6 \pm 16.5$ & $36.1 \pm 117.3$ & $176.6 \pm 166.4$ \\
stock & $43.7 \pm 7.5$ & $49.2 \pm 12.5$ & $86.4 \pm 18.3$ & $441.9 \pm 93.4$ \\
triazines & $2.7 \pm 1.3$ & $5.7 \pm 4.2$ & $12.3 \pm 7.9$ & $79.9 \pm 47.9$ \\
wisconsin & $2.2 \pm 2.9$ & $0.8 \pm 0.7$ & $1.6 \pm 1.0$ & $12.1 \pm 8.8$ \\
\hline
\end{tabular}

Table 2: Average number of splitter nodes, estimated using $10 \times 10$-fold cross-validation

\begin{tabular}{l|cc|cc}
\hline Dataset & Outer CV & Inner CV & Outer CV & Inner CV \\
& \multicolumn{2}{|c|}{$(\lambda=1)$} & \multicolumn{2}{c}{$(\lambda=0.5)$} \\
\hline 2dplanes & $1.0291 \pm 0.0227$ & $1.0325 \pm 0.0116$ & $1.0154 \pm 0.0136$ & $1.0165 \pm 0.0050$ \\
abalone & $2.1765 \pm 0.1322$ & $2.1760 \pm 0.0175$ & $2.1860 \pm 0.1489$ & $2.1883 \pm 0.0223$ \\
ailerons & $0.0002 \pm 0.0000$ & $0.0002 \pm 0.0000$ & $0.0002 \pm 0.0000$ & $0.0002 \pm 0.0000$ \\
autoMpg & $2.8265 \pm 0.4974$ & $2.9106 \pm 0.1146$ & $2.8838 \pm 0.7388$ & $2.8713 \pm 0.1031$ \\
auto-price & $2345.9 \pm 947.96$ & $2375.7 \pm 206.05$ & $2095.4 \pm 934.33$ & $2102.4 \pm 197.13$ \\
bank32nh & $0.0826 \pm 0.0035$ & $0.0827 \pm 0.0005$ & $0.0822 \pm 0.0034$ & $0.0823 \pm 0.0005$ \\
bank8FM & $0.0303 \pm 0.0010$ & $0.0304 \pm 0.0002$ & $0.0296 \pm 0.0009$ & $0.0297 \pm 0.0002$ \\
cal-housing & $49426 . \pm 2117.7$ & $49654 . \pm 441.43$ & $49207 . \pm 2648.1$ & $49052 . \pm 404.43$ \\
cpu-act & $2.4352 \pm 0.2096$ & $2.4376 \pm 0.0617$ & $2.5078 \pm 1.0848$ & $2.3680 \pm 0.0454$ \\
cpu-small & $2.8872 \pm 0.1708$ & $2.8882 \pm 0.0717$ & $2.8713 \pm 0.7162$ & $2.8271 \pm 0.0475$ \\
delta-ailerons & $0.0002 \pm 0.0000$ & $0.0002 \pm 0.0000$ & $0.0002 \pm 0.0000$ & $0.0002 \pm 0.0000$ \\
delta-elevators & $0.0014 \pm 0.0000$ & $0.0014 \pm 0.0000$ & $0.0014 \pm 0.0000$ & $0.0014 \pm 0.0000$ \\
diabetes-numeric & $0.6591 \pm 0.2513$ & $0.6092 \pm 0.0575$ & $0.5936 \pm 0.2268$ & $0.5876 \pm 0.0358$ \\
elevators & $0.0022 \pm 0.0001$ & $0.0022 \pm 0.0000$ & $0.0021 \pm 0.0001$ & $0.0021 \pm 0.0000$ \\
fried & $1.0105 \pm 0.0118$ & $1.0117 \pm 0.0021$ & $1.0086 \pm 0.0110$ & $1.0093 \pm 0.0014$ \\
house-16H & $37361 . \pm 7601.3$ & $35647 . \pm 545.93$ & $35609 . \pm 3811.1$ & $35180 . \pm 590.30$ \\
house-8L & $31724 . \pm 2056.8$ & $31486 . \pm 350.37$ & $31277 . \pm 1990.9$ & $31170 . \pm 367.68$ \\
housing & $5.5683 \pm 4.6668$ & $4.3045 \pm 0.3692$ & $5.2818 \pm 3.4914$ & $4.6027 \pm 0.5248$ \\
kin8nm & $0.1170 \pm 0.0034$ & $0.1182 \pm 0.0011$ & $0.1091 \pm 0.0031$ & $0.1109 \pm 0.0009$ \\
machine-cpu & $63.466 \pm 29.309$ & $65.233 \pm 9.6004$ & $49.436 \pm 26.409$ & $53.887 \pm 7.8728$ \\
mv & $0.0064 \pm 0.0019$ & $0.0065 \pm 0.0008$ & $0.0615 \pm 0.3890$ & $0.0235 \pm 0.0055$ \\
pol & $9.6527 \pm 2.2639$ & $9.3432 \pm 0.4544$ & $8.0089 \pm 2.3251$ & $7.6774 \pm 0.3870$ \\
puma32H & $0.0204 \pm 0.0055$ & $0.0213 \pm 0.0022$ & $0.0135 \pm 0.0076$ & $0.0155 \pm 0.0037$ \\
puma8NH & $3.1872 \pm 0.0844$ & $3.1876 \pm 0.0114$ & $3.2019 \pm 0.0828$ & $3.2029 \pm 0.0105$ \\
pyrim & $0.1261 \pm 0.0748$ & $0.1208 \pm 0.0159$ & $0.1046 \pm 0.0679$ & $0.1109 \pm 0.0160$ \\
servo & $0.4964 \pm 0.3067$ & $0.5413 \pm 0.1118$ & $0.4875 \pm 0.2905$ & $0.5298 \pm 0.0978$ \\
stock & $1.0138 \pm 0.2016$ & $1.0119 \pm 0.0645$ & $0.8645 \pm 0.1234$ & $0.8878 \pm 0.0460$ \\
triazines & $0.1503 \pm 0.0401$ & $0.1449 \pm 0.0088$ & $0.1370 \pm 0.0379$ & $0.1348 \pm 0.0083$ \\
wisconsin & $34.482 \pm 4.1926$ & $34.045 \pm 0.8672$ & $33.486 \pm 4.0019$ & $33.388 \pm 0.6931$ \\
\hline
\end{tabular}

Table 3: Root mean squared error obtained using outer $10 \times 10$-fold cross-validation, for AMT ( $\lambda=1$ and $\lambda=0.5$ ), vs. average root mean squared error of the same model in inner 10-fold cross-validation 
is a multiple linear regression model ${ }^{3}$

It is also interesting to compare the cross-validation estimates obtained using inner cross-validation on the training data, encountered when selecting the "best" number of iterations to perform, with those established from the outer $10 \times 10$-fold cross-validation used to obtain an unbiased error estimate. Table 3 shows this for $\lambda=1$ and $\lambda=0.5$, based on root mean squared error rather than root relative squared error. Note that the estimates from the inner cross-validation runs are averages over the $10 \times 10$-fold outer cross-validation runs, one for each train/test split occurring in the outer cross-validation. Thus, they are averages obtained from 1,000 train/test splits of the full data. As can be seen, the estimates are quite close: the average estimate obtained using inner cross-validation is always well within one standard deviation of the estimate from the outer crossvalidation. This means that when building an alternating model tree with IterativeClassifierOptimizer in practice, the cross-validation estimate associated with the number of iteration it chooses can be used as an indicator of performance on new data. This is helpful because no separate, outer evaluation process is necessary to get an indication of error on fresh data.

\section{CONCLUSIONS}

Model trees have proven to be a useful technique in practical regression problems. In this paper, we present alternating model trees grown using forward stagewise additive modeling and show that they achieve significantly lower squared error than $\mathrm{M}^{\prime}$ model trees on several regression problems.

In contrast to alternating decision trees for classification, our alternating model trees have prediction nodes containing simple linear regression models. To reduce computational complexity and data fragmentation when constructing splitter nodes, splits are taken to be the median value of an appropriately chosen attribute. Moreover, shrinkage is used to combat overfitting.

The size of the tree depends on the number of iterations of forward stagewise additive modeling that are performed. In our experiments, we have chosen this parameter using internal cross-validation. Our results indicate that the performance estimate obtained in this manner is close to the actual performance for all datasets considered in our experiments. Therefore, no external cross-validation run is required in order to establish an estimate of expected performance on fresh data.

\section{References}

[1] K. Bache and M. Lichman. UCI machine learning repository, 2013.

[2] B. Bhattacharya and D. P. Solomatine. Neural networks and M5 model trees in modelling water level-discharge relationship. Neurocomputing, 63:381-396, 2005.

[3] W. Buntine. Learning classification trees. Statistics and Computing, 2(2):63-73, 1992.

[4] A. Etemad-Shahidi and J. Mahjoobi. Comparison between M5 model tree and neural networks for prediction of significant wave height in Lake Superior. Ocean Engineering, 36(15):1175-1181, 2009.

\footnotetext{
${ }^{3}$ But not the least-squares multiple linear regression model.
}

[5] Y. Freund and L. Mason. The alternating decision tree learning algorithm. In Proc 16th International Conf on Machine Learning, pages 124-133, San Francisco, CA, USA, 1999. Morgan Kaufmann Publishers Inc.

[6] J. Friedman, T. Hastie, and R. Tibshirani. Additive logistic regression: a statistical view of boosting. The Annals of Statistics, 28(2):337-407, 042000.

[7] M. Hall, E. Frank, G. Holmes, B. Pfahringer, P. Reutemann, and I. H. Witten. The WEKA data mining software: an update. SIGKDD Explorations, 11(1):10-18, 2009 .

[8] G. Holmes, B. Pfahringer, R. Kirkby, E. Frank, and M. Hall. Multiclass alternating decision trees. In Proc 13th European Conf on Machine Learning, Helsinki, Finland, pages 161-172. Springer, 2002.

[9] R. Kohavi and C. Kunz. Option decision trees with majority votes. In Proc 14th International Conf on Machine Learning, pages 161-169, San Francisco, CA, USA, 1997. Morgan Kaufmann Publishers Inc.

[10] D. Lubinsky. Tree structured interpretable regression. In D. Fisher and H.-J. Lenz, editors, Learning from Data, volume 112 of Lecture Notes in Statistics, pages 387-398. Springer New York, 1996.

[11] D. Malerba, F. Esposito, M. Ceci, and A. Appice. Topdown induction of model trees with regression and splitting nodes. IEEE Transactions on Pattern Analysis and Machine Intelligence, 26(5):612-625, 2004.

[12] C. Nadeau and Y. Bengio. Inference for the generalization error. Machine Learning, 52(3):239-281, 2003.

[13] E. Ould-Ahmed-Vall, J. Woodlee, C. Yount, K. A. Doshi, and S. Abraham. Using model trees for computer architecture performance analysis of software applications. In IEEE International Symposium on Performance Analysis of Systems $\&$ Software, 200\%. ISPASS 2007., pages 116-125. IEEE, 2007.

[14] M. Pal and S. Deswal. M5 model tree based modelling of reference evapotranspiration. Hydrological Processes, 23(10):1437-1443, 2009 .

[15] J. R. Quinlan. Learning with continuous classes. In Proc 5th Australian Joint Conf on Artificial Intelligence, pages 343-348, Singapore, 1992. World Scientific.

[16] D. P. Solomatine and K. N. Dulal. Model trees as an alternative to neural networks in rainfall-runoff modelling. Hydrological Sciences Journal, 48(3):399-411, 2003.

[17] M. Taghi Sattari, M. Pal, H. Apaydin, and F. Ozturk. M5 model tree application in daily river flow forecasting in Sohu Stream, Turkey. Water Resources, 40(3):233$242,2013$.

[18] Y. Wang and I. H. Witten. Inducing model trees for continuous classes. In Proc 9th European Conf on Machine Learning Poster Papers, pages 128-137, 1997. 